\title{
Imagerie tridimensionnelle et chirurgie orale : planifier pour simplifier
}

\section{Bodard AG, Fabris M, Vere-Goulet S, Dessouter A. \\ (Centre Léon Bérard, Lyon)}

Le recours au scanner $X$ est fréquent pour les chirurgies orales complexes (avulsion de dents incluses, kystectomies, chirurgie orthodontique, implantologie), lorsqu'il existe un risque anatomique pour les organes nobles adjacents. Si le recours aux guides chirurgicaux associés aux reconstitutions tridimensionnelles a été largement décrit en implantologie, il reste encore peu développé en chirurgie orale.

L'analyse des coupes scanner ne permet pas toujours la représentation mentale des rapports des éléments anatomiques entre eux. Les reconstructions tridimensionnelles affranchissent le clinicien de cette étape préopératoire. La reconstitution tridimensionnelle surfacique à partir des acquisitions scanographiques (MVS ${ }^{\circledR}$, Hospices civils de Lyon et $3 \mathrm{DNe} 0^{\circledR}, 3 \mathrm{DNeovision}$ Lyon - France) permet, en chirurgie avulsive, de limiter les complications postopératoires notamment nerveuses. En cas de nécessité d'ostectomie, l'importance de celleci peut être déterminée en préopératoire. L'intérêt d'une reconstruction surfacique autorise la segmentation de chaque élément anatomique de manière isolée, celui-ci pouvant être manipulé indépendamment.

Associée à la conception d'un guide stéréolithographique grâce au recalage des empreintes optiques des modèles sur les images scanner, on peut ainsi déterminer avec précision les axes chirurgicaux, et réaliser une chirurgie guidée, devenant ainsi minimalement invasif. Ceci prend tout son intérêt lors de lésions au contact du nerf mandibulaire ou à proximité du sinus maxillaire.

En chirurgie à visée orthodontique, cette technologie permet de déterminer la voie d'abord de la dent incluse en cas de désinclusion avant traction. Ainsi, il est possible de déterminer un couloir d'accès par voie vestibulaire pour une canine incluse palatine, simplifiant grandement la procédure et l'accès au site opératoire. II est aisé de choisir précisément le positionnement des minivis d'ancrage, en collaboration avec l'orthodontiste. L'analyse des images permet de mesurer si l'espace ouvert par l'orthodontiste est suffisant pour accueillir la dent à tracter ou s'il persiste un obstacle mécanique. Cela permet également de mieux mettre en évidence les éventuelles zones d'ankylose avant d'effectuer le collage et la traction.

Différents cas cliniques illustrent les applications de la reconstruction tridimensionnelle surfacique en chirurgie orale au sein du Service de Consultations et traitements Dentaires des Hospices Civils de Lyon.

BODARD Anne Gaëlle anne-gaelle.bodard@lyon.unicancer.fr 